\title{
Canadian Space Astronomy: past, present and future (Presentation Video)
}

\section{John Hutchings}

John B. Hutchings, "Canadian Space Astronomy: past, present and future (Presentation Video)," Proc. SPIE 9143, Space Telescopes and Instrumentation 2014: Optical, Infrared, and Millimeter Wave, 91431H (22 June 2014); doi: 10.1117/12.2063485

Event: SPIE Astronomical Telescopes + Instrumentation, 2014, Montréal, Quebec, Canada 


\title{
Canadian Space Astronomy: Past, Present and Future (Presentation Video)
}

\author{
John B. Hutchings, NRC - Herzberg Institute of Astrophysics (Canada)
}

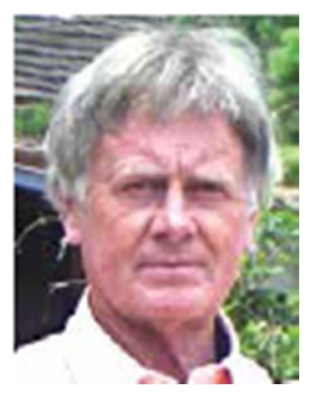

\begin{abstract}
Canadian astronomers have participated in space astronomy since the first OAO missions in the 1960s and 1970s. Individual Canadian scientists have been members of HST instrument teams, and advisory groups for IUE and HEAO missions, as well as competing successfully for observing time on NASA, ESA, and Japanese astronomy satellites. With the formation of the Canadian Space Agency, Canada became partner in the FUSE mission, the ISRO Astrosat, and the JWST, providing hardware and science team membership. The Canadian Astronomy Data centre was one of the three original world-wide archive distribution centres for HST, and now is involved in many space and ground-based data services. The MOST observatory is an all- Canadian microsat that has operated for nearly a decade. Canada is currently involved in partnership in a number of imminent space facilities, as well as participating in teams defining future missions. I will describe this history, and review the technical and scientific capability that exist in Canada now. I will outline prospects for the future, including a concept for a high resolution orbiting telescope that will fill the gap in high resolution UV astronomy when HST operations cease.
\end{abstract}

View presentation video on SPIE's Digital Library: http://dx.doi.org/10.1117/12.2063485.3664756653001 